\title{
Impressum, Vol. 11, Supplement 1, 1988
}

\section{S. Karger}

Verlag für Medizin und Naturwissenschaften GmbH, Postfach 1724, D-8034 Germering

Presserechtlich verantwortlich: Walter Kunz, Gesellschafter

Wissenschaftlicher Beirat

H. W. Bauer, Berlin; W. Bossnev, Sofia/Bulgarien; H. Denck, Wien; V. Diehl, Köln; P. Drings, Heidelberg; E. Dühmke, Göttin-gen; S. Eckhardt, Budapest; H. Ehrhart, München; H. H. Fiebig, Freiburg; W. M. Gallmeier, Nürnberg; E. H. Graul, Marburg/Lahn; R. Hartenstein, München; H. Heimpel, Ulm; K. P. Hellriegel, Berlin; Ch. Herfarth, Heidelberg; H. W. von Heyden, Einbeck; D. Hoelzer, Frankfurt/M.; J. H. Holzner, Wien; R. Hünig, Basel; W. Hunstein, Heidelberg; H. J. Illiger, Oldenburg; U. R. Kleeberg, Hamburg; H. O. Klein, Köln; B. Kornhuber, Frankfurt/M.; H. Löfñer, Kiel; U. Mohr, Hannover; K. Munk, Heidelberg; G. A. Nagel, Göttingen; J. P. Obrecht, Basel; A. Pñeiderer, Freiburg; K. Possinger, München; W. Queißer, Mannheim; H. Riehm, Hannover; J. Roth, Basel; E. Scherer, Essen; M. Schroder, Göttingen; S. Seeber, Leverkusen; H. J. Senn, St. Gallen; A. Stacher, Wien; V. Sturm, Heidelberg; St. Tanneberger, Berlin/DDR; W. Vahlensieck, Bonn; H. D. Waller, Tubingen; M. Wannenmacher, Freiburg; W. Wilmanns, München; K. Wilms, Würzburg; K. zum Winkel, Heidelberg; H. Wrba, Wien.

Schriftleitung: S. Eckhardt, Budapest J. H. Holzner, Wien G. A. Nagel, Göttingen Anzeigen

S. Karger Verlag für Medizin und Naturwissenschaften GmbH, Postfach 1724, D-8034 Germering, Telefon (089) 844021, Telefax (089) 8418083.

Für den Inhalt außerhalb des redaktionellen Teiles (insbesondere Anzeigen, Industrieinformationen, Pressezitate und Kongreßinfor-mationen) übernehmen Herausgeber und Verlag keine Gewähr.

Eine Markenbezeichnung kann warenzeichenrechtlich geschützt sein, auch wenn bei ihrer Verwendung in dieser Zeitschrift das Zeichen ${ }^{\circledR}$ oder ein anderer Hinweis auf etwa bestehende Schutz-rechte fehlen sollte.

Alle Rechte, insbesondere das Recht der Vervielfältigung und Mikrokopie sowie der Übersetzung, vorbehalten. Nachdruck, auch auszugsweise, nur mit Genehmigung des Verlages. (C) Copyright 1988 by S. Karger Verlag für Medizin und Naturwissenschaften GmbH, Postfach 1724, D-8034 Germering ISBN 3-8055-4935-0

Satz und Druck: Gebr. Parcus KG, D-8000 München 34

Redaktionskomitee dieses Sonderheftes

H. Ehrhart U. Fink H. Graeff C. Kullmann R. Kürzl E. Kuß H. Lindner W. Meier H. Merkl G. Ries P. Scheidel L. Schmid

G. Schubert-Fritschle

H. Schünemann

R. Schröck

G. Sondershaus

G. Stein 
J. C. de Waal

K. Zellmann

Ladenpreis dieses Sonderheftes DM 18,- incl. MwSt.; Abonnenten erhalten es unberechnet. Bezugspreis der Zeitschrift:

Für Jahrgang 11, 1988, DM 148 -/öS 1036,-/SFr. 116.-1 Einzelheft kostet DM 28 -/öS 196 /SFr. 22.-, einschließlich MwSt., zuzüglich Postgebühren. 